\title{
Prognostic Value of Plasma HER2 Gene Copy Number in HER2-Positive Metastatic Breast Cancer Treated with First-Line Trastuzumab
}

This article was published in the following Dove Press journal: OncoTargets and Therapy

\author{
$\operatorname{Ran} \operatorname{Ran}^{1, *}$ \\ Wenfa Huang ${ }^{1} *$ \\ Yaxin Liul,* \\ Lin Shao ${ }^{2}$ \\ Xiaoran Liu' \\ Yunyun $\mathrm{Niu}^{2}$ \\ Weiyao Kong' \\ Shiping $\mathrm{Bo}^{2}$ \\ Hope S Rugo ${ }^{3}$ \\ Sijia $L^{2}$ \\ Huiping Li $\mathbb{D}^{1}$ \\ 'Key Laboratory of Carcinogenesis and \\ Translational Research (Ministry of \\ Education/Beijing), Department of Breast \\ Oncology, Peking University Cancer \\ Hospital and Institute, Beijing I00I42, \\ People's Republic of China; ${ }^{2}$ Department \\ of Clinical Research, Yikon Genomics Co. \\ Ltd., Shanghai, People's Republic of China; \\ ${ }^{3}$ University of California San Francisco \\ Helen Diller Family Comprehensive \\ Cancer Center, San Francisco, CA, USA \\ *These authors contributed equally to \\ this work
}

Correspondence: Huiping Li;

Hope S Rugo

Tel +86-10-88|96380

Email huipingli2012@hotmail.com;

Hope.Rugo@ucsf.edu
Objective: Patients with HER2-positive metastatic breast cancer (MBC) benefit from trastuzumab-based therapy but eventually develop intrinsic or acquired resistance. Whether plasma HER2 gene copy number $(\mathrm{GCN})$ could predict survival after trastuzumab treatment remained controversial. We evaluated the prognostic value of plasma HER2 GCN using lowcoverage whole-genome sequencing (LC-WGS).

Methods: The plasma was collected from HER2-positive MBC patients whose pre-therapeutic samples were available before first-line trastuzumab-based treatment. Plasma DNA was extracted and assessed by LC-WGS for HER2 GCN. The optimal cut-off point for HER2 GCN to shorter survival was determined by receiver operating characteristic (ROC) curve analysis.

Results: A total of 49 patients were retrieved from 2013 to 2017, among whom 21 had multiple organ involvement ( $\geq 3$ sites). Variations of HER2 GCN in pre-therapeutic plasma ranged from 1.89 to 23.86 (median $=2.59$ ). ROC analysis identified the optimal cut-off point for HER2 GCN as $2.82(P=0.005)$, with 23 patients had high-level HER2 GCN and 26 in the low-level group. Both progression-free survival (PFS, $P=0.032$ ) and overall survival (OS, $P=0.006$ ) were adversely associated with high-level HER2 GCN. In multivariate analyses, high HER2 GCN was independently associated with shorter PFS [hazard ratio $(\mathrm{HR})=2.042, P=0.037$, while both high HER2 GCN $(\mathrm{HR}=4.909, P=0.004)$ and more metastatic organs $(\mathrm{HR}=4.019, P=0.011)$ were negative prognostic factors for OS.

Conclusion: In this population of patients with HER2-positive MBC, individuals with high HER2 GCNs in plasma had worse prognosis after trastuzumab-based therapy. Plasma HER2 GCN may be a prognostic marker in these patients.

Keywords: gene copy number, HER2-positive, metastatic breast cancer, whole genome sequencing, plasma

\section{Background}

Human epidermal growth factor receptor 2 (HER2) is overexpressed or amplified in approximately $20-30 \%$ of invasive breast cancer (BC) cases. ${ }^{1}$ HER2 overexpression leads to increased tumor proliferation, more aggressive tumor behavior, and poor prognosis, whereas qualifies patients for anti-HER2 treatment. ${ }^{2,3}$ The combination of monoclonal antibody trastuzumab (and more recent, combination with pertuzumab) with chemotherapy has been shown to improve the progression-free survival (PFS) and overall survival (OS) in patients with metastatic breast cancer (MBC), and to reduce the risk of recurrence and improve survival in patients with early-stage disease. ${ }^{3-5}$ 
However, despite the established benefit of trastuzumabbased therapy for patients with HER2-positive (HER2+) MBC, a subset of patients do not respond to the combined treatment and have poor survival. ${ }^{3,4}$ Understanding the biologic and clinical variables that predicting resistance is complex and remains problematic in clinical practice. Several alternative HER2-targeted treatment options exist, with new and effective therapies in development. ${ }^{6-8}$ Identifying patients less likely to respond to standard first-line therapy would allow the evaluation of alternative therapeutic options earlier in the treatment course. ${ }^{7-9}$

HER2 + BC harbors wide variations in HER2 gene copy number (GCN), and high levels of HER2 gene amplification prove to be associated with worse prognosis in some studies, representing a potential factor for the differing responses to the combination therapy with trastuzumab and chemotherapy. ${ }^{10-14}$ Interestingly, the HER2 status of primary tumors and metastases are not always concordant. ${ }^{15}$ Furthermore, the availability of metastatic tumor tissue is limited, and repeated biopsies are impractical. ${ }^{16}$ Thus, the clinical application of circulating tumor DNA (ctDNA), a non-invasive "liquid biopsy", provides the opportunity for serial sequencing and analysis. ${ }^{9,16,17}$ Measurement of genomic mutations in ctDNA has been suggested to have prognostic value in several cancer types, such as colorectal cancer, malignant melanoma, and breast cancer. ${ }^{17-19}$

However, little is currently known about the impact of different levels of plasma HER2 amplification on prognosis and response to trastuzumab-based therapy. Multiple studies have focused on the clinical significance of HER2 GCN in the ctDNA of BC patients, but the results were controversial owing to their small scale or the relatively low accuracy of the detection methods used, such as droplet digital PCR (ddPCR). ${ }^{16,20-22}$ Therefore, we evaluated the amplification of HER2 GCN in the plasma using low-coverage whole-genome sequencing (LC-WGS) and studied its prognostic role in HER2+ MBC patients.

\section{Materials and Methods Study Design}

Patients with HER2 + MBC who had pre-therapeutic plasma samples were consecutively retrieved from our medical record system between June 2013 and June 2017. The included patients should meet all of the following criteria: (1) histologically confirmed of HER2 + BC with recurrent or de novo metastatic disease; (2) received trastuzumab-based therapy (with either chemotherapy or endocrine therapy) as first-line treatments; (3) had measurable disease according to the Response Evaluation Criteria in Solid Tumors criteria (RECIST) version 1.1. ${ }^{23}$ Patients were excluded if they had clinically significant cardiac dysfunction, other types of malignant diseases, or an Eastern Cooperative Oncology Group (ECOG) performance status of $>2$.

The plasma samples were collected from patients with written informed consent for their samples to be used in the future. The research protocol was approved by the Medical Ethics Committee of Peking University Cancer Hospital (No. 2016KT92), and conducted in accordance with the reporting recommendations for tumor marker prognostic studies (REMARK) guidelines. ${ }^{24}$

The clinicopathological characteristics and the response to drug treatment, evaluated by computed tomography every two or three cycles of treatment and categorized as complete response (CR), partial response (PR), stable disease (SD), or progressive disease (PD) according to the RECIST 1.1 criteria, were extracted from their medical records. Raw survival data were obtained from the patients' attending physician or through phone-call follow-up every six months. PFS was defined as the time from the start of trastuzumab-based therapy to first disease progression or death, and event-free patients were censored at last follow-up. OS was measured from disease recurrence or metastasis until death from any cause or last contact.

\section{Blood Sample Processing}

Whole blood $(4 \mathrm{~mL})$ was collected in the EDTA tubes and then centrifuged at $1600 \mathrm{~g}$ for $10 \mathrm{~min}$ at $4^{\circ} \mathrm{C}$. The supernatant was then transferred to a fresh tube and centrifuged for a second time at $16,000 \mathrm{~g}$ for $10 \mathrm{~min}$ at $4^{\circ} \mathrm{C}$. The plasma was temporarily stored at $-80^{\circ} \mathrm{C}$ until DNA extraction. At least $5 \mathrm{ng}$ of the ctDNA extracted from $500 \mu \mathrm{L}$ of plasma for each case was subjected to generate LC-WGS library as previously described. ${ }^{25,26}$ Sequencing was performed on an Illumina HiSeq 2500 sequencer (Illumina, San Diego, CA, USA). The quality of library was checked by quantitative-PCR and Bioanalyzer (Agilent, USA). Approximately 5 million sequencing reads were obtained from each sample.

\section{Data Analysis and HER2 GCN Calculation}

The ctDNA data were analyzed as previously described by an observer who was blinded to all clinical data. ${ }^{25,26}$ 
Briefly, the raw were demultiplexed and converted to FASTQ format in Illumina Consensus Assessment of Sequence and Variation (CASAVA, version 1.8.2) software. The low-quality bases and adapters were removed from the FASTQ file using Trimmomatic (version 0.35). High-quality reads were mapped to the hg19 reference genome using BWA (version 0.7.12-r1039) and unique mapped reads were extracted. The reference genome was divided into non-overlapping observation windows (bins) of $1 \mathrm{Mb} / 200 \mathrm{~Kb}$ in size. The read number and guaninecytosine (GC) content in each bin were computed. GC bias correction was performed for each $1 \%$ GC content. The bin read count was normalized by GC content and a reference dataset using $\mathrm{R}$ (version 3.00) to represent the relative copy number of HER2 gene.

\section{Statistical Analysis}

All statistical analyses were performed using SPSS software (version 19.0) and GraphPad Prism (version 7.0). To assess whether an increased HER2 GCN affected treatment efficacy of trastuzumab-based therapy, we defined patients with progression or death within 24 weeks as the poor survival group, and the others as good survival group. A receiver operating characteristic (ROC) curve was used to evaluate the prediction performance of plasma HER2 GCN, and Youden index was calculated to determine the optimal cutoff points.

Pearson's $\chi^{2}$ test and Fisher's exact test were performed to determine the relationship between HER2 GCN and clinicopathological parameters as appropriate. Survival curves based on HER2 GCN were estimated using the Kaplan-Meier survival analysis and compared by Log-rank test. Univariate and multivariate analyses were performed using Cox proportional hazards model with backward elimination method based on the likelihood ratio test. Variables with a $P$ value of $>0.10$ in the multivariate analyses were removed. All tests were twotailed, with a $P$ value of $<0.05$ considered statistically significant.

\section{Results}

\section{Patients' Characteristics}

In total, 49 consecutive female patients who had pre-therapeutic plasma were included. Clinicopathological characteristics of the study population are summarized in Table 1 . The median age of the included patients at diagnosis was
50 years (range, 29-79 years). Seven of 49 (14.3\%) patients were initially diagnosed with MBC, and 42 (85.7\%) experienced recurrence or metastatic disease after surgery, among whom, 14 (33.3\%) patients underwent re-biopsy and HER2

Table I Clinicopathological Characteristics of the HER2-Positive Metastatic Breast Cancer $(n=49)$

\begin{tabular}{|c|c|}
\hline Characteristic & Number (\%) \\
\hline \multicolumn{2}{|l|}{ Age (years) } \\
\hline Median (range) & $50(29-79)$ \\
\hline$<50$ & $24(49.0)$ \\
\hline$\geq 50$ & $25(51.0)$ \\
\hline \multicolumn{2}{|l|}{ Menopausal status } \\
\hline Premenopausal & $15(30.6)$ \\
\hline Postmenopausal & $34(69.4)$ \\
\hline \multicolumn{2}{|l|}{ ECOG score } \\
\hline 0 & $43(87.8)$ \\
\hline $\mathrm{I}-2$ & $6(12.2)$ \\
\hline \multicolumn{2}{|l|}{ Hormone-receptor status } \\
\hline ER and/or PR positive & $31(63.3)$ \\
\hline ER and PR negative & $18(36.7)$ \\
\hline \multicolumn{2}{|l|}{ Neo/adjuvant trastuzumab therapy } \\
\hline Yes & $8(16.3)$ \\
\hline No & $4 \mid(83.7)$ \\
\hline \multicolumn{2}{|l|}{ Disease status at treatment } \\
\hline Relapsed & $42(85.7)$ \\
\hline Initially metastatic & $7(14.3)$ \\
\hline \multicolumn{2}{|l|}{ Metastasis organs } \\
\hline Skin or chest wall & $18(36.7)$ \\
\hline Lymph node & $37(75.5)$ \\
\hline Bone & $20(40.8)$ \\
\hline Lung & $14(28.6)$ \\
\hline Liver & $20(40.8)$ \\
\hline Brain & I (2.0) \\
\hline Others $^{\dagger}$ & II (22.4) \\
\hline \multicolumn{2}{|l|}{ Visceral metastasis } \\
\hline Yes & $29(59.2)$ \\
\hline No & $20(40.8)$ \\
\hline \multicolumn{2}{|l|}{ Number of metastatic organs } \\
\hline$<3$ sites & $28(57.1)$ \\
\hline$\geq 3$ sites & $21(42.9)$ \\
\hline \multicolumn{2}{|l|}{ Concomitant agents } \\
\hline Taxanes alone & $28(57.1)$ \\
\hline Taxane-based & $10(20.4)$ \\
\hline Others & II (22.5) \\
\hline
\end{tabular}

Note: ${ }^{\dagger}$ Others include pleura, peritoneum, ovary, or pericardium.

Abbreviation: ECOG, Eastern Cooperative Oncology Group. 
conversion from negative to positive was observed in one patient (Case 12 in Table S1). Thirty-one (63.3\%) patients were hormone-receptor positive, while visceral metastases were identified in 29 (59.2\%) patients. Most of the patients (77.6\%) received taxanes as the concomitant agents administrated with trastuzumab at first-line.

A

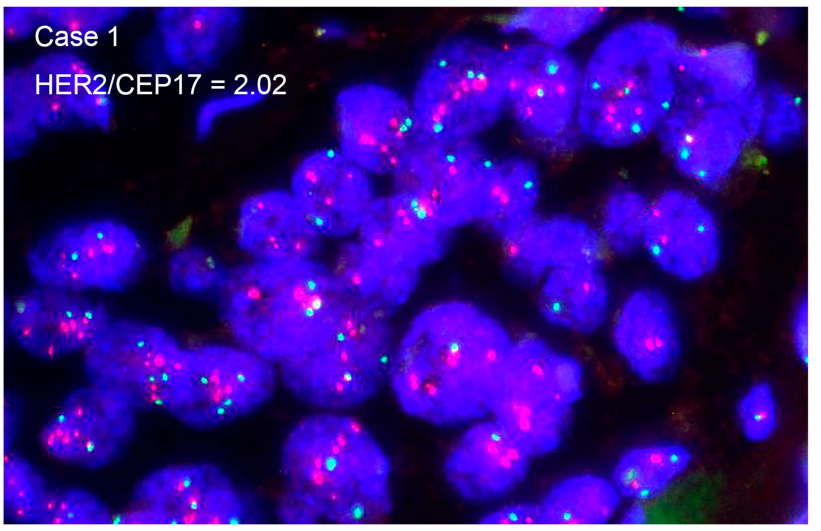

B

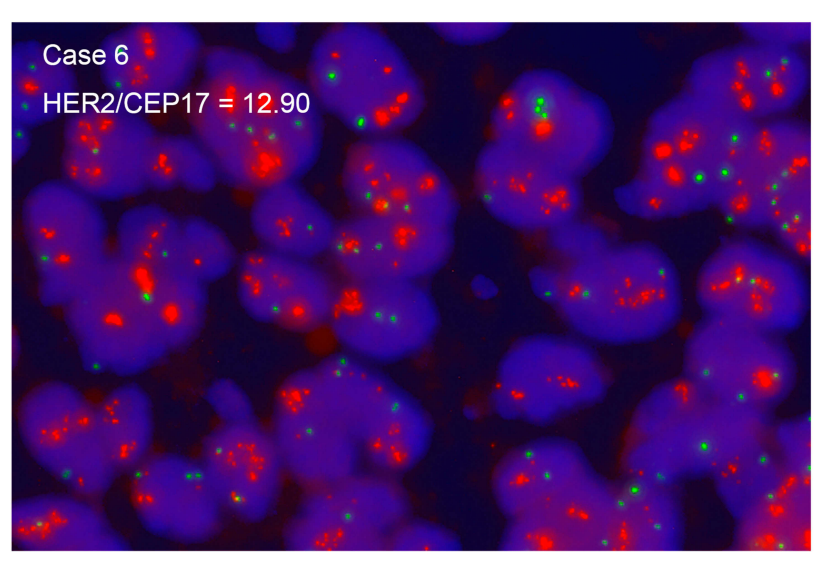

C

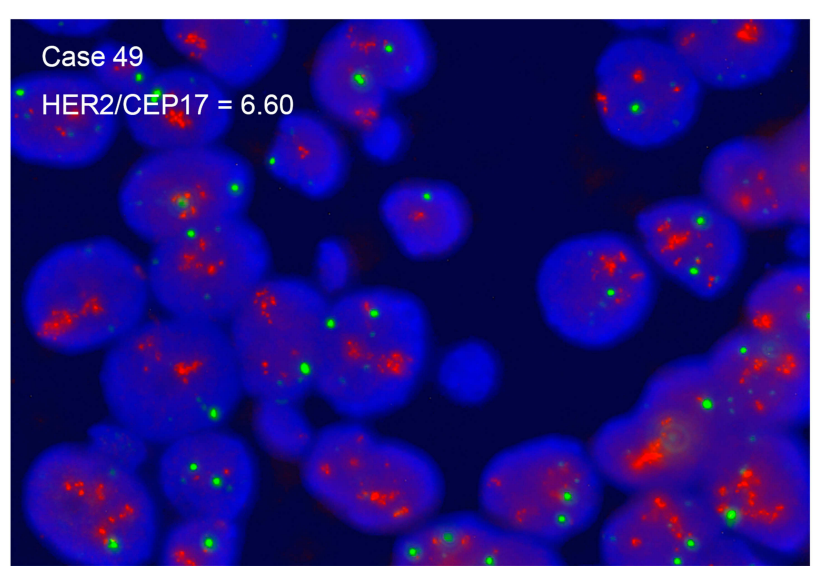

\section{Plasma HER2 GCN and Clinical}

\section{Characteristics}

The GCN of HER2 in the plasma collected just before the start of treatment is presented in Table S1 and Figure 1 (median 2.59, range 1.89-23.86). ROC analysis was performed to determine the optimal cut-off points that

D

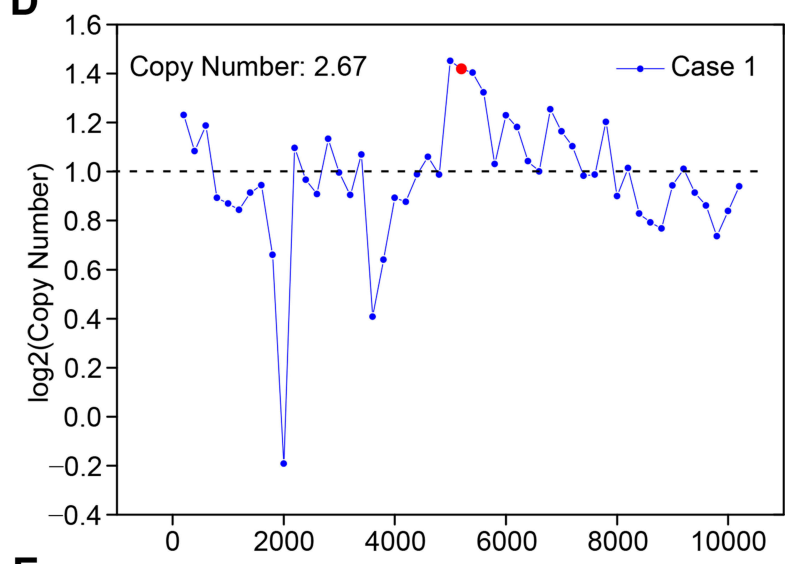

E

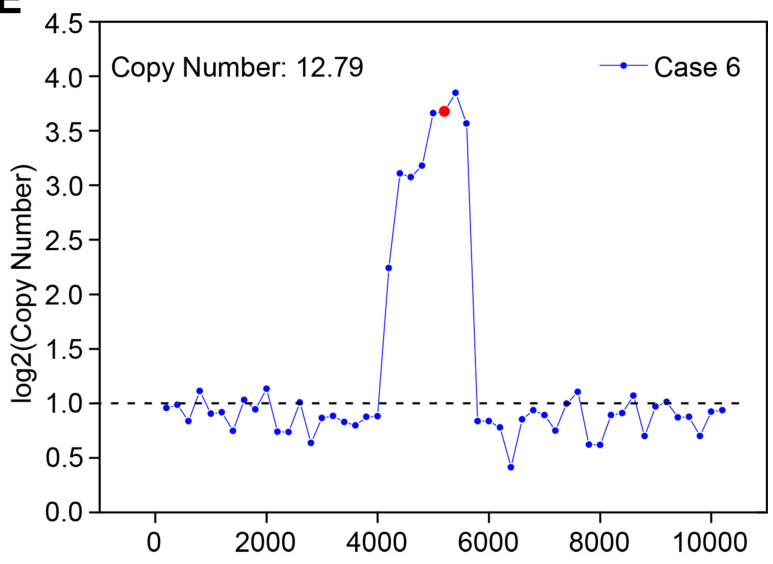

$\mathbf{F}$

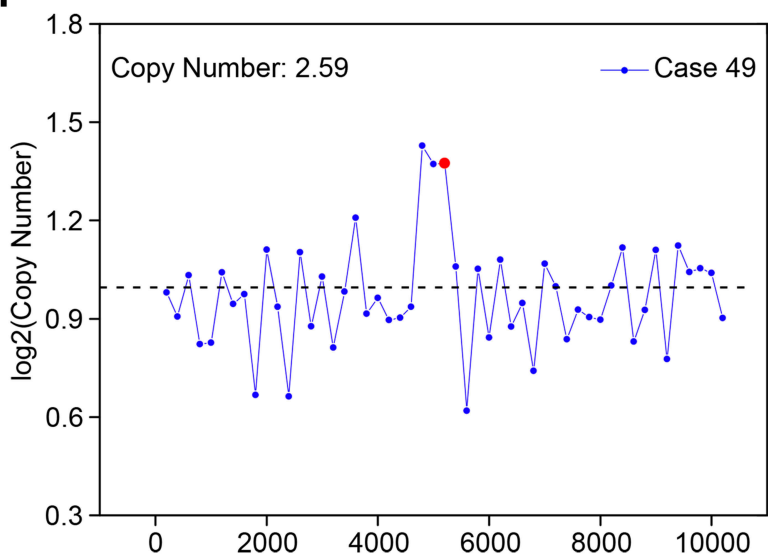

Figure I Comparison of HER2 gene amplification in tumor tissues by FISH (A-C, primary or metastatic, original magnification $\times 1000)$ and corresponding plasma by NGS (D-F). The $x$-axis (D-F) shows the 10,000 kb region adjacent to HER2, with each dot representing the gene copy number (GCN) of each $200 \mathrm{~kb}$ segment. The enlarged red dot denotes the HER2 gene locus. (A-F) HER2 amplification was identified in both the tumor tissues and plasma samples of cases I, 6, and 49, but HER2 GCN in the metastatic plasma $(\mathbf{F})$ of case 49 was lower than in the primary tumor tissues $(\mathbf{C})$. 
distinguished patients with shorter survival. As shown in Figure 2, the AUC of ROC curve was 0.758 [95\% confidence interval (CI) $0.606-0.911 ; P=0.005]$, and the optimal cut-off point for HER2 GCN was 2.82. The relationship of plasma HER2 GCN with various clinicopathological factors is presented in Table 2. Of the patients with higher levels of HER2 GCN (HER2-high, >2.82), 69.6\% (16/23) developed more than three organ metastases compared with $19.2 \%(5 / 26)$ patients with low-level HER2 GCN (HER2-low, $P<0.001$ ). In addition, there were also significant differences in aspects of liver metastasis (56.5\% vs $26.9 \%, P=0.035)$ and bone metastasis $(65.2 \%$ vs $18.5 \% ; P=0.001)$.

\section{Baseline HER2 GCN and Patient Survival}

The follow-up was performed until August 2019, with a median follow-up time of 29.0 months (range, 5.3-75.7 months). During the follow-up period, disease progression was observed in 35 patients ( $71.4 \%$ ), and 21 patients (42.9\%) died. The tumor response to trastuzumab-based therapy is summarized in Table 2, and more patients in the HER2-high group developed PD at the first efficacy evaluation $(26.1 \%$ vs $3.9 \%$ ). Kaplan-Meier survival analysis showed that patients in HER2-high group had significantly shorter PFS (median PFS, 6.6 months vs 24.8 months; $P=0.032$ ), compared with

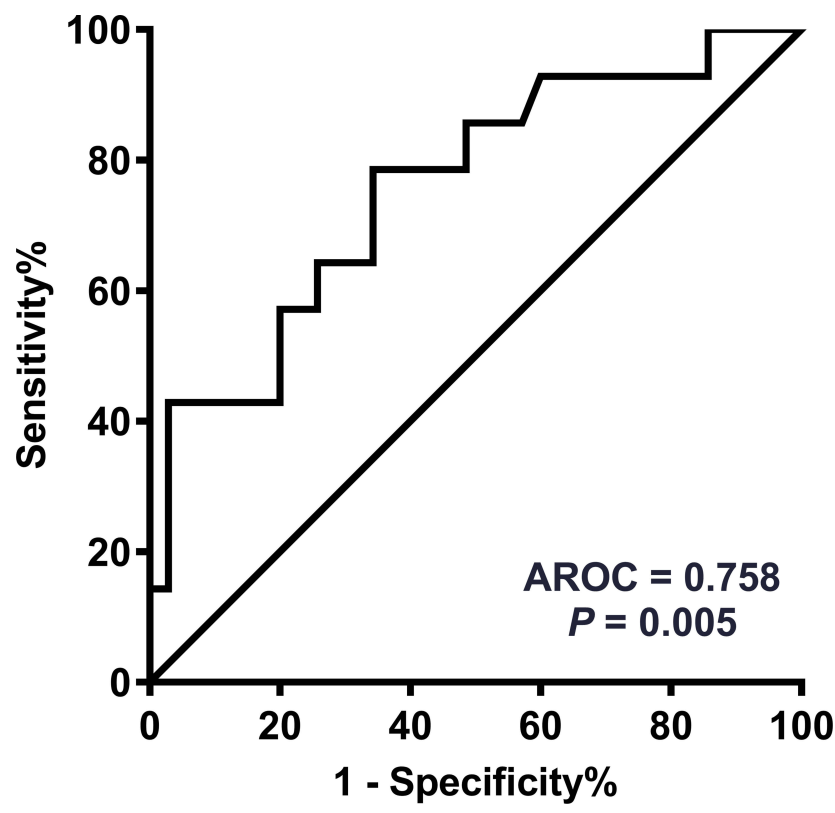

Figure 2 Optimal cut-off points determined by receiver operator characteristic (ROC) curve. Plasma HER2 GCN of 2.82 was identified as the optimal cut-off point for distinguishing patients with worse prognosis.
Table 2 Clinicopathological Characteristics and HER2 GCN Status $(n=49)$

\begin{tabular}{|c|c|c|c|}
\hline \multirow[t]{2}{*}{ Characteristic } & \multicolumn{2}{|c|}{ HER2 GCN [N (\%)] } & \multirow{2}{*}{$\begin{array}{l}P \\
\text { value }\end{array}$} \\
\hline & $\begin{array}{l}\leq 2.82 \\
(n=26)\end{array}$ & $\begin{array}{l}>2.82 \\
(n=23)\end{array}$ & \\
\hline $\begin{array}{l}\text { Age (years) } \\
\quad<50 \\
\quad \geq 50\end{array}$ & $\begin{array}{l}\text { I5 (57.7) } \\
\text { II (42.3) }\end{array}$ & $\begin{array}{l}9(39.1) \\
14(60.9)\end{array}$ & 0.195 \\
\hline $\begin{array}{c}\text { Menopausal status } \\
\text { Premenopausal } \\
\text { Postmenopausal }\end{array}$ & $\begin{array}{l}6(23.1) \\
20(76.9)\end{array}$ & $\begin{array}{l}9(39.1) \\
14(60.9)\end{array}$ & 0.224 \\
\hline $\begin{array}{l}\text { ECOG score } \\
\qquad \begin{array}{l}0 \\
I-2\end{array}\end{array}$ & $\begin{array}{l}25(96.2) \\
I(3.8)\end{array}$ & $\begin{array}{l}18(78.3) \\
5(21.7)\end{array}$ & 0.141 \\
\hline $\begin{array}{l}\text { Hormone-receptor status } \\
\text { ER and/or PR positive } \\
\text { ER and PR negative }\end{array}$ & $\begin{array}{l}19(73.1) \\
7(26.9)\end{array}$ & $\begin{array}{l}\text { I2 (52.2) } \\
\text { II (47.8) }\end{array}$ & 0.130 \\
\hline $\begin{array}{l}\text { Neo/adjuvant trastuzumab } \\
\text { therapy } \\
\text { Yes } \\
\text { No }\end{array}$ & $\begin{array}{l}4(15.4) \\
22(84.6)\end{array}$ & $\begin{array}{l}4(17.4) \\
19(82.6)\end{array}$ & 1.000 \\
\hline $\begin{array}{l}\text { Metastasis organs } \\
\text { Skin or chest wall } \\
\text { Lymph node } \\
\text { Bone } \\
\text { Lung } \\
\text { Liver } \\
\text { Brain } \\
\text { Others }\end{array}$ & $\begin{array}{l}7(26.9) \\
18(69.2) \\
5(19.2) \\
8(30.8) \\
7(26.9) \\
0 \\
3(11.5)\end{array}$ & $\begin{array}{l}\text { II (47.8) } \\
\text { I9 (82.6) } \\
\text { I5 (65.2) } \\
6(26.1) \\
\text { I3 (56.5) } \\
\text { I (4.3) } \\
8(34.8)\end{array}$ & $\begin{array}{l}0.130 \\
0.277 \\
0.00 I^{*} \\
0.717 \\
0.035^{*} \\
\mathrm{NE} \\
0.085\end{array}$ \\
\hline $\begin{array}{l}\text { Visceral metastasis } \\
\text { Yes } \\
\text { No }\end{array}$ & $\begin{array}{l}14(53.8) \\
12(46.2)\end{array}$ & $\begin{array}{l}15(65.2) \\
8(34.8)\end{array}$ & 0.419 \\
\hline $\begin{array}{l}\text { Number of metastatic } \\
\text { organs } \\
\quad<3 \text { sites } \\
\geq 3 \text { sites }\end{array}$ & $\begin{array}{l}21(80.8) \\
5(19.2)\end{array}$ & $\begin{array}{l}7(30.4) \\
16(69.6)\end{array}$ & $<0.001 *$ \\
\hline $\begin{array}{l}\text { Concomitant agents } \\
\text { Taxanes alone } \\
\text { Taxane-based } \\
\text { Others }\end{array}$ & $\begin{array}{l}15(57.7) \\
5(19.2) \\
6(23.1)\end{array}$ & $\begin{array}{l}13(56.6) \\
5(21.7) \\
5(21.7)\end{array}$ & 0.975 \\
\hline $\begin{array}{l}\text { Tumor response } \\
\text { Complete response } \\
\text { Partial response } \\
\text { Stable disease } \\
\text { Progressive disease }\end{array}$ & $\begin{array}{l}\text { I (3.9) } \\
\text { I5 (57.7) } \\
9(34.6) \\
\text { I (3.9) }\end{array}$ & $\begin{array}{l}0 \\
12(52.2) \\
5(21.7) \\
6(26.1)\end{array}$ & \\
\hline
\end{tabular}

Note: *Significant difference at $\alpha$ value of 0.05 .

Abbreviations: ECOG, Eastern Cooperative Oncology Group; NE, not estimable. 
HER2-low patients (Figure 3). Similarly, OS (median OS, 27.6 months vs not estimable; $P=0.006$ ) was also significantly shorter in HER2-high patients.

Cox analyses were performed to evaluate the effects of pre-therapeutic HER2 GCN on the PFS and OS of HER2+ MBC patients (Table 3). Univariate analyses revealed that HER2-high patients exhibited an association with shortened PFS [hazard ratio $(\mathrm{HR})=2.042$, 95\% CI 1.045 $-3.991 ; P=0.037]$ and $\mathrm{OS}(\mathrm{HR}=3.468,95 \%$ CI 1.355 $-8.877 ; P=0.009$ ) (Table 3 ). When adjusting for hormone-receptor status, history of neo/adjuvant trastuzumab therapy, and metastatic lesions, HER2 GCN proved to be the only independent negative prognostic factor for PFS $(\mathrm{HR}=2.042,95 \%$ CI $1.045-3.991 ; P=0.037)$. As for OS, however, both high-level HER2 GCN (HR $=4.909$, 95\% CI 1.684-14.305, $P=0.004)$ and more organ metastases $(\geq 3, \mathrm{HR}=4.019,95 \%$ CI 1.369-11.803, $P=0.011)$ were proved to be related with worse OS.

\section{Dynamics of Plasma HER2 GCN During} Trastuzumab-Based Treatment

The surveillance value of plasma HER2 GCN for trastuzumab-based therapy was investigated in 21 patients, and a total of 36 plasma samples were analyzed. Changes in the levels of HER2 GCN were compared with the performance of radiographic measurements according to RECIST criteria (v1.1). Eleven out of 21 candidates were identified to be HER2-high at baseline. In those whose plasma was available at $\geq 3$ time points, most patients exhibited a decrease in HER2 GCN when benefiting from treatment, with increased HER2 GCN concurring with (case 7 and case 22) or even preluding (case 10, case 19, and case 28) disease progression (Figure 4A). Otherwise, fluctuations of HER2 GCN in HER2-low patients were not predominant, with the exception of Case 27 who had a notable increase of HER2 GCN at disease progression (Figure 4B).

\section{Discussion}

HER2-positive MBC is incurable but can be treated with the extensive use of trastuzumab. ${ }^{4}$ Traditionally, patients with HER2 overexpression as detected by IHC or gene amplification as determined by FISH can derive substantial benefit from trastuzumab-based therapy. ${ }^{21}$ However, several studies suggest that clinical outcomes after trastuzumab-based therapy still vary among different levels of HER2 GCN. ${ }^{12,27}$ Due to the critical role of HER2 status for tailoring the therapeutic management of MBC patients, we designed a noninvasive and repeatable method for serial quantification of HER2 GCN in plasma samples based on the LC-WGS technique and verified its clinical implication in prognosis prediction. ${ }^{25,26}$

We retrospectively assessed 49 HER2+ MBC patients who had pre-therapeutic plasma in our center and observed that patients with high-level HER2 GCN (>2.82) were associated with worse prognosis even after trastuzumabbased therapy. In multivariate analyses, high-level HER2 GCN proved to be an independent negative factor for PFS; whereas, interpreting its value on OS needed to be tempered, as these patients seemed to have more organ involvement, and both of them were associated with shorter survival. The specific mechanisms accounting for the unfavorable prognosis for HER2-high patients remain unknown. $^{28} \mathrm{~A}$ previous study reported that patients with an incremental gain in HER2 GCN in metastatic tissue had
A

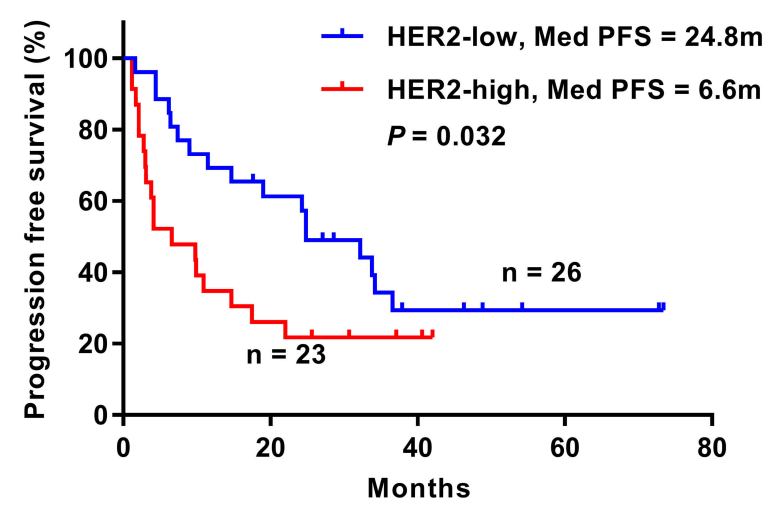

B

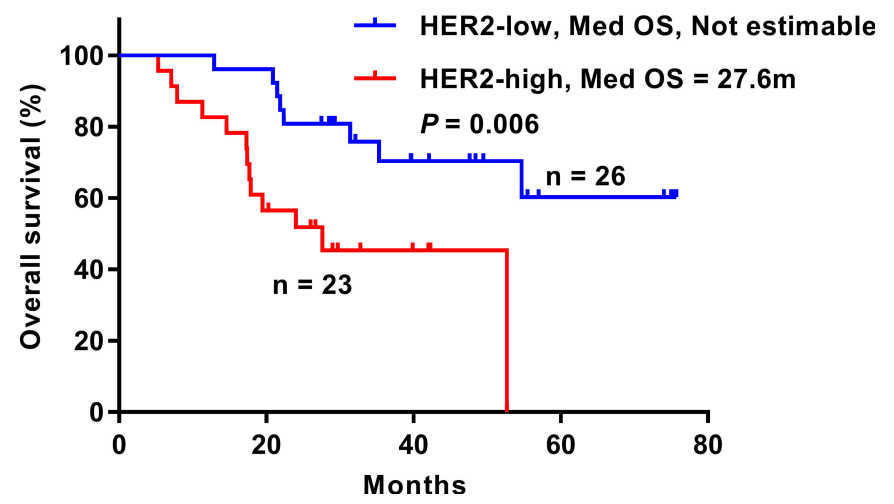

Figure 3 Kaplan-Meier survival curve for progression-free survival and overall survival according to baseline HER2 gene copy number (GCN). (A) PFS was significantly shorter in HER2-high patients. (B) OS was significantly shorter in HER2-high patients. HER2-low, HER2 GCN of $\leq 2.82$; HER2-high, HER2 GCN of >2.82. 


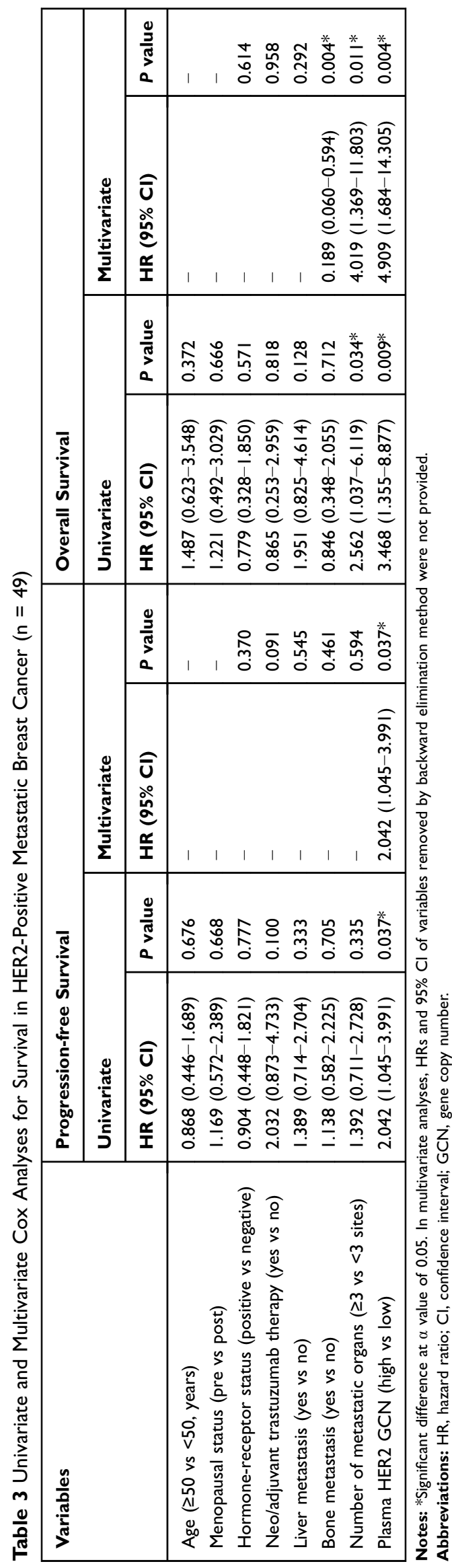

worse outcomes even after trastuzumab-based therapy, which implied that the increase in HER2 GCN during tumor progression may predict an intrinsically more aggressive disease. ${ }^{29,30}$ In a cohort of MBC patients, Bates et al also identified a subset with high HER2 expression associated with trastuzumab resistant and shorter survival. ${ }^{31}$ Thus, we speculate that the continuous expression and over-crowding of HER2 protein on the cytomembrane, caused by the high-level HER2 amplification, might increase the crosstalk with other receptor and extensive activation of the downstream signal. ${ }^{27}$ And the anti-HER2 therapy based on trastuzumab and chemotherapy maybe not enough for patients with multiple metastasis site and high-HER2 GCN. ${ }^{5}$

Our results were concordant with several studies with respect to the impact of tumor tissue HER2 GCN on patients' prognosis. ${ }^{27,30,32}$ In the adjuvant setting, Xuan et al quantified HER2 GCN in 291 HER2+ BC patients and identified a subgroup of HER2-high patients with poor prognosis. ${ }^{27} \mathrm{~A}$ recent analysis in 278 patients with early breast cancer also demonstrated that patients whose tumors revealed high HER2/CEP17 ratios $(>8)$ exhibited impaired survival. ${ }^{33}$ Moreover, several compelling evidence also suggested that HER2 GCN levels can predict treatment response to trastuzumab. Subgroup analyses of both the HERA and N9831 trial supported the notion that patients with high HER2 GCN might fail to benefit from adjuvant trastuzumab treatment. ${ }^{12,34}$ In MBC, previous studies also reported a trend of shorter survival in patients with high-level HER2 amplification, and an increase in HER2 GCN from primary tumors to metastasis was associated with shorter OS. ${ }^{10,30}$ However, the role of HER2 GCN seemed to be undetermined in the metastatic setting, as some studies in which FISH data were obtained only from primary tumor tissues showed opposite results. ${ }^{35-37}$

Serial ctDNA tracking makes it possible to timely detect HER2 GCN in the metastatic setting and to reasonably forecast the clinical outcomes. Guan et al measured the HER2 GCN in ctDNA of 105 patients based on LC-WGS, similar to that of our study, found a high concordant rate of $86.5 \%$ (sensitivity, 77.1\%; specificity, 95.8\%) compared with primary tumor tissues, which surpassed those of previous results. ${ }^{26}$ And in the 41 patients, they revealed a positive relationship between high-level HER $2 \mathrm{GCN}(\geq 4)$ and PR rate to multi-line chemotherapy. In our present study, the ROC analysis was applied, and 2.82 was the optimal cut-off that discriminated between patients with good and poor outcomes. Our results, however, indicated that high-level 


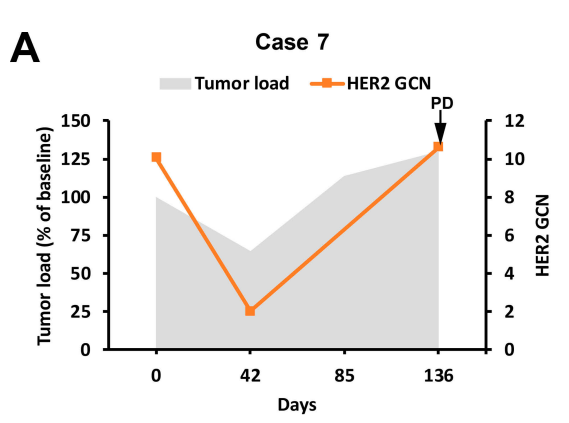

Case 19

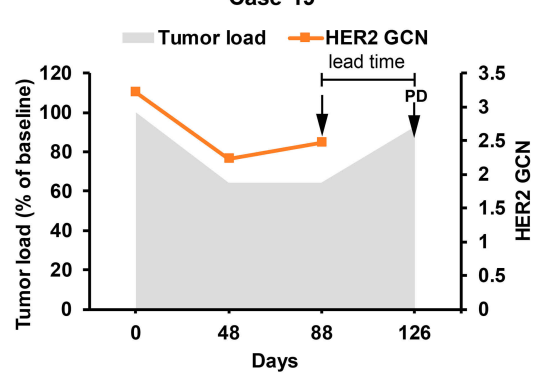

B

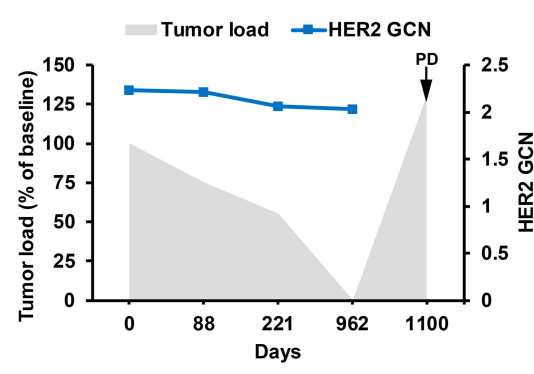

Case 24

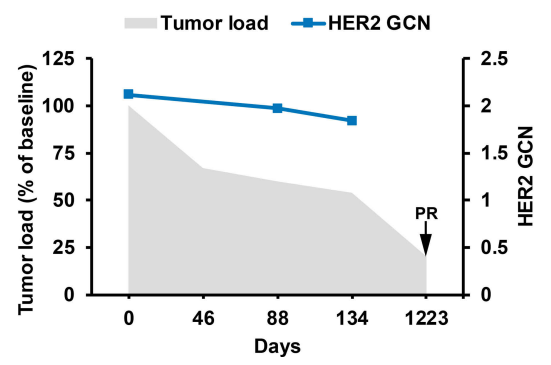

Case 10

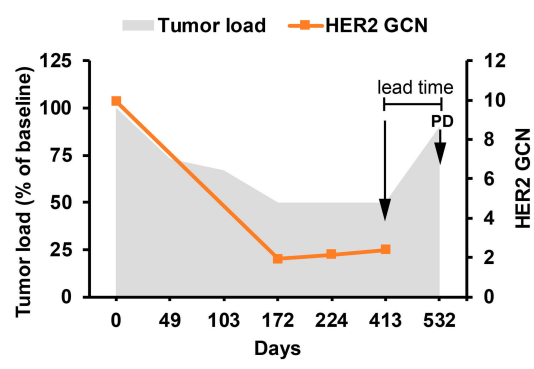

Case 22

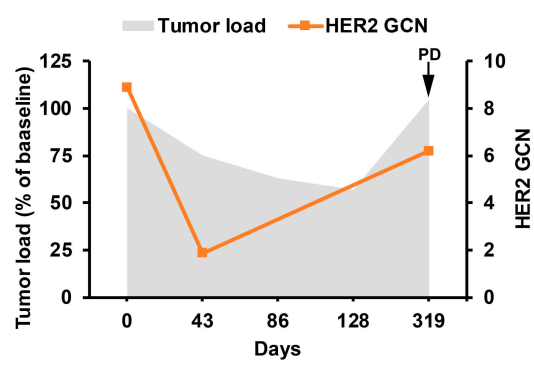

Case 13

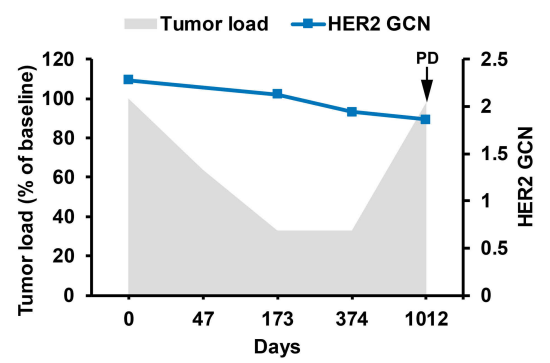

Case 27

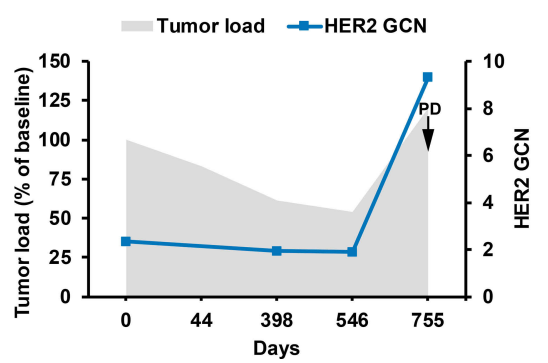

Case 16

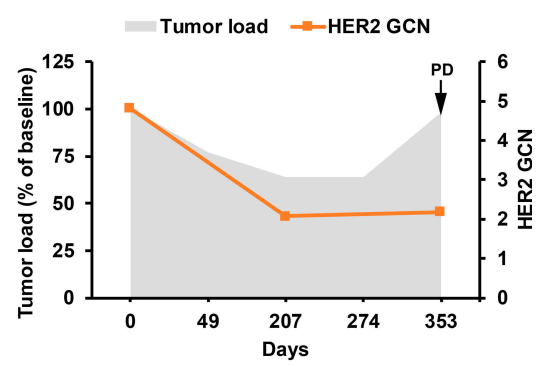

Case 28

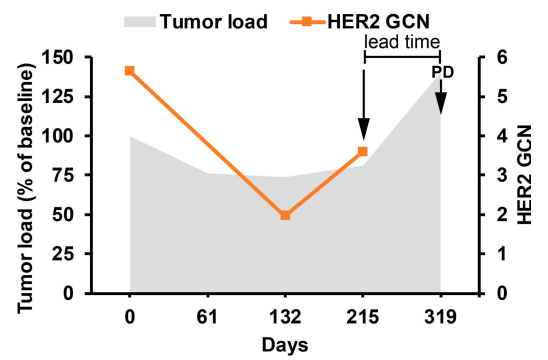

Case 17

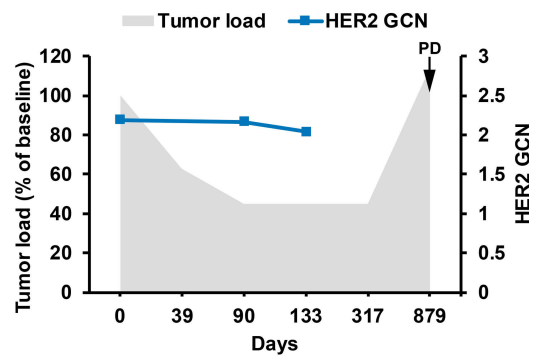

Figure 4 Dynamic changes of plasma HER2 gene copy number (GCN) in response to trastuzumab-based therapy. (A) Changes in HER2 GCN in six HER2-high patients who had three or more blood samples. Levels of HER2 GCN decreased in response to treatment. Notable increase in HER2 GCN and tumor load was concurrently detected in case 7 and case 22, while case 10, case 19, and case 28 had an increased GCN preluding disease progression. (B) Changes in HER2 GCN were not predominant in HER2-low patients, except for case 27 who exhibited an increase of HER2 GCN at disease progression.

Abbreviations: PR, partial response; PD, progressive disease.

HER2 GCN was a negative prognostic factor for both PFS and OS after trastuzumab-based therapy. Moreover, HER2high patients were associated with more organ metastases, and the sequential HER2 GCN seemed to decrease first and then increase again, which was concurrent with trastuzumab benefit and disease progression, respectively. Previous studies have demonstrated the value of ctDNA longitudinal analysis on monitoring the tumor response and it was correlated with the changes in tumor burden. ${ }^{9,38}$ Although the study by Sorensen et al failed to observe the prognostic value of baseline HER2 amplification, they revealed a reduction in HER2 GCN that was correlated with favorable benefit from trastuzumab-based therapy. ${ }^{21}$ Other former studies on HER2 amplification using NGS also suggested its association with the effects of trastuzumab emtansine (T-DM1) and pyrotinib. ${ }^{16,22}$ 
Unlike HER2 GCN tracking in ctDNA, the potential clinical significance of serum HER2 extracellular domain (ECD) has been investigated for two decades. ${ }^{39}$ HER2 ECD can be released into the blood via alternative initiation of translation or proteolytic cleavage, but its correlation with the status of tissue HER2 is not prominent. ${ }^{40}$ The cut-off value of elevated HER2 ECD is recommended to be $\geq 15 \mathrm{ng} /$ $\mathrm{mL}$, and it has been proposed as a promising biomarker for patients' prognosis and for monitoring the disease status in HER2+ BC patients, similar to HER2 GCN in ctDNA. ${ }^{41}$ However, the use of serum HER2 ECD to guide adjuvant therapy or to predict trastuzumab benefit was not recommended by the American Society of Clinical Oncology, because the evidence was not strong enough. ${ }^{42}$

We acknowledge that our study has some limitations due to the inherent characteristic of retrospective data collection and its small sample size. First, the dynamic samples were obtained at various time points, and only in half of patients. However, the values of HER2 GCN remain stable when the patients continue to achieve treatment benefit (Figure 4), implying that samples obtained in a period of the same disease status may have similar clinical significance. But integrated analyses of plasma HER2 GCN at regular time points are imperative in evaluating the prognostic and monitoring value of serial HER2 GCN sequencing. Second, a comparison of HER 2 GCN values between metastatic tumor tissues and plasma was lacking, because HER2 gene amplification by FISH was not available for all metastatic patients in this retrospective study. Finally, a training set that validated our findings was lacking in this study, because of the limited sample size. A large-scale, prospective study will be performed to validate our findings in the future. And furthermore, ROC analysis has been performed to determine the optimal cut-off that distinguished patients with shorter survival, which could avoid the introduction of artificial bias. Despite these limitations, our data and those of others imply the clinical value of HER2 GCN analysis in MBC patients.

\section{Conclusions}

In conclusion, the present study showed that patients with high HER2 GCN (>2.82) in pre-therapeutic plasma may have worse survival after first-line trastuzumab-based therapy in our HER2+ MBC cohort. Therefore, analysis of plasma HER 2 GCN by LC-WGS may have a potential value in prognosis predicting after trastuzumab-based treatment, and serve as a complement, apart from the conventional tissue HER2 GCN analysis and imaging assessment, for a more effective management of HER2+ MBC in first line.

\section{Ethics Approval and Informed Consent}

All procedures performed in studies involving human participants were in accordance with the ethical standards of the Medical Ethics Committee of Peking University Cancer Hospital (No. 2016KT92) and with the 1964 Helsinki declaration and its later amendments or comparable ethical standards. Informed consent was obtained from all individual participants included in the study.

\section{Acknowledgments}

We thank H. Nikki March, PhD, from Liwen Bianji, Edanz Editing China, for editing the English text of a draft of this manuscript. The abstract of this paper was presented at the 2018 ASCO annual meeting as an online publication with interim findings. The abstract was published in "Poster Abstracts" in Journal of Clinical Oncology. 2018. 36 (15):e13018 [https://ascopubs.org/doi/abs/10.1200/JCO. 2018.36.15_suppl.e13018].

\section{Author Contributions}

All authors made substantial contributions to conception and design, acquisition of data, or analysis and interpretation of data; took part in drafting the article or revising it critically for important intellectual content; gave final approval of the version to be published; and agree to be accountable for all aspects of the work.

\section{Funding}

This study was funded by the National Natural Science Foundation of China (Grant No. 81502269).

\section{Disclosure}

Lin Shao, Yunyun Niu, Shiping Bo, and Sijia Lu are employees of Yikon Genomics Co. Ltd., Shanghai, China. Dr Hope S Rugo reports grants from Pfizer, Merck, Novartis, Lilly, Genentech, OBI Pharma, Odonate, Daiichi, Eisai, Seattle Genetics, Macrogenics, and Immunomedics, during the conduct of the study; and travel support from Daiichi, Mylan, Pfizer, Amgen, Merck, AstraZeneca, Macrogenics, and Puma. The authors report no other conflicts of interest in this work.

\section{References}

1. Slamon DJ, Clark GM, Wong SG, Levin WJ, Ullrich A, McGuire WL. Human breast cancer: correlation of relapse and survival with amplification of the HER-2/neu oncogene. Science. 1987;235(4785):177182. doi:10.1126/science. 3798106 
2. Krishnamurti U, Silverman JF. HER2 in breast cancer: a review and update. Adv Anat Pathol. 2014;21(2):100-107. doi:10.1097/PAP.000 0000000000015

3. Li H, Shao B, Yan Y, et al. Efficacy and safety of trastuzumab combined with chemotherapy for first-line treatment and beyond progression of HER2-overexpressing advanced breast cancer. Chin J Cancer Res. 2016;28(3):330-338. doi:10.21147/j.issn.1000-9604.2016.03.07

4. Slamon DJ, Leyland-Jones B, Shak S, et al. Use of chemotherapy plus a monoclonal antibody against HER2 for metastatic breast cancer that overexpresses HER2. N Engl J Med. 2001;344(11):783792. doi:10.1056/NEJM200103153441101

5. Baselga J, Cortes J, Kim SB, et al. Pertuzumab plus trastuzumab plus docetaxel for metastatic breast cancer. $N$ Engl J Med. 2012;366 (2):109-119. doi:10.1056/NEJMoa1113216

6. Geyer CE, Forster J, Lindquist D, et al. Lapatinib plus capecitabine for HER2-positive advanced breast cancer. $N$ Engl J Med. 2006;355 (26):2733-2743. doi:10.1056/NEJMoa064320

7. Verma S, Miles D, Gianni L, et al. Trastuzumab emtansine for HER2positive advanced breast cancer. N Engl J Med. 2012;367(19):17831791. doi:10.1056/NEJMoa1209124

8. Ma F, Li Q, Chen S, et al. Phase I study and biomarker analysis of pyrotinib, a novel irreversible Pan-ErbB receptor tyrosine kinase inhibitor, in patients with human epidermal growth factor receptor 2-positive metastatic breast cancer. J Clin Oncol. 2017;35(27):31053112. doi:10.1200/JCO.2016.69.6179

9. Dawson S-J, Tsui DWY, Murtaza M, et al. Analysis of circulating tumor DNA to monitor metastatic breast cancer. $N$ Engl J Med. 2013;368(13):1199-1209. doi:10.1056/NEJMoa1213261

10. Gullo G, Bettio D, Torri V, Masci G, Salvini P, Santoro A. Level of HER2/neu gene amplification as a predictive factor of response to trastuzumab-based therapy in patients with HER2-positive metastatic breast cancer. Invest New Drugs. 2009;27(2):179-183. doi:10.1007/ s10637-008-9155-y

11. Borley A, Mercer T, Morgan M, et al. Impact of HER2 copy number in IHC2+/FISH-amplified breast cancer on outcome of adjuvant trastuzumab treatment in a large UK cancer network. Br J Cancer. 2014;110(8):2139-2143. doi:10.1038/bjc.2014.147

12. Dowsett M, Procter M, McCaskill-Stevens W, et al. Disease-free survival according to degree of HER2 amplification for patients treated with adjuvant chemotherapy with or without 1 year of trastuzumab: the HERA trial. J Clin Oncol. 2009;27(18):2962-2969. doi:10.1200/JCO.2008.19.7939

13. Tubbs R, Barlow WE, Budd GT, et al. Outcome of patients with early-stage breast cancer treated with doxorubicin-based adjuvant chemotherapy as a function of HER2 and TOP2A status. J Clin Oncol. 2009;27(24):3881-3886. doi:10.1200/JCO.2008.20.1566

14. Veeraraghavan J, De Angelis C, Mao R, et al. A combinatorial biomarker predicts pathologic complete response to neoadjuvant lapatinib and trastuzumab without chemotherapy in patients with HER2+ breast cancer. Ann Oncol. 2019;30(6):927-933. doi:10. 1093/annonc/mdz076

15. Schrijver W, Suijkerbuijk KPM, van Gils CH, van der Wall E, Moelans CB, van Diest PJ. Receptor conversion in distant breast cancer metastases: a systematic review and meta-analysis. J Natl Cancer Inst. 2018;110(6):568-580. doi:10.1093/jnci/djx273

16. Ma F, Zhu W, Guan Y, et al. ctDNA dynamics: a novel indicator to track resistance in metastatic breast cancer treated with anti-HER2 therapy. Oncotarget. 2016;7(40):66020-66031. doi:10.18632/oncotarget.11791

17. Li H, Xu Y, Zhao F, et al. Plasma PIK3CA ctDNA specific mutation detected by next generation sequencing is associated with clinical outcome in advanced breast cancer. Am J Cancer Res. 2018;8 (9):1873-1886.

18. Gabriel E, Bagaria SP. Assessing the impact of circulating tumor DNA (ctDNA) in patients with colorectal cancer: separating fact from fiction. Front Oncol. 2018;8:297. doi:10.3389/fonc.2018.00297
19. Ashida A, Sakaizawa K, Uhara H, Okuyama R. Circulating tumour DNA for monitoring treatment response to Anti-PD-1 immunotherapy in melanoma patients. Acta Derm Venereol. 2017;97(10):12121218. doi: $10.2340 / 00015555-2748$

20. Bechmann T, Andersen RF, Pallisgaard N, et al. Plasma HER2 amplification in cell-free DNA during neoadjuvant chemotherapy in breast cancer. J Cancer Res Clin Oncol. 2013;139(6):995-1003. doi:10.1007/s00432-013-1413-5

21. Sorensen BS, Mortensen LS, Andersen J, Nexo E. Circulating HER2 DNA after trastuzumab treatment predicts survival and response in breast cancer. Anticancer Res. 2010;30(6):2463-2468.

22. Sakai H, Tsurutani J, Iwasa T, et al. HER2 genomic amplification in circulating tumor DNA and estrogen receptor positivity predict primary resistance to trastuzumab emtansine (T-DM1) in patients with HER2-positive metastatic breast cancer. Breast Cancer. 2018;25 (5):605-613. doi:10.1007/s12282-018-0861-9

23. Eisenhauer EA, Therasse P, Bogaerts J, et al. New response evaluation criteria in solid tumours: revised RECIST guideline (version 1.1). Eur J Cancer. 2009;45(2):228-247. doi:10.1016/j.ejca.2008. 10.026

24. McShane LM, Altman DG, Sauerbrei W, Taube SE, Gion M, Clark GM. REporting recommendations for tumor MARKer prognostic studies (REMARK). Breast Cancer Res Treat. 2006;100(2):229235. doi:10.1007/s10549-006-9242-8

25. Wang H, Li B, Liu Z, et al. HER2 copy number of circulating tumour DNA functions as a biomarker to predict and monitor trastuzumab efficacy in advanced gastric cancer. Eur J Cancer. 2018;88:92-100. doi:10.1016/j.ejca.2017.10.032

26. Guan X, Liu B, Niu Y, et al. Longitudinal HER2 amplification tracked in circulating tumor DNA for therapeutic effect monitoring and prognostic evaluation in patients with breast cancer. Breast. 2020;49:261-266. doi:10.1016/j.breast.2019.12.010

27. Xuan Q, Ji H, Tao X, Xu Y, Zhang Q. Quantitative assessment of HER2 amplification in HER2-positive breast cancer: its association with clinical outcomes. Breast Cancer Res Treat. 2015;150(3):581588. doi:10.1007/s10549-015-3334-2

28. De P, Hasmann M, Leyland-Jones B. Molecular determinants of trastuzumab efficacy: what is their clinical relevance? Cancer Treat Rev. 2013;39(8):925-934. doi:10.1016/j.ctrv.2013.02.006

29. Yao J, Weremowicz S, Feng B, et al. Combined cDNA array comparative genomic hybridization and serial analysis of gene expression analysis of breast tumor progression. Cancer Res. 2006;66(8):40654078. doi:10.1158/0008-5472.CAN-05-4083

30. Gullo G, Bettio D, Zuradelli M, et al. Level of HER2/neu amplification in primary tumours and metastases in HER2-positive breast cancer and survival after trastuzumab therapy. Breast. 2013;22 (2):190-193. doi:10.1016/j.breast.2013.01.005

31. Bates M, Sperinde J, Kostler WJ, et al. Identification of a subpopulation of metastatic breast cancer patients with very high HER2 expression levels and possible resistance to trastuzumab. Ann Oncol. 2011;22(9):2014-2020. doi:10.1093/annonc/mdq706

32. Adamczyk A, Kruczak A, Harazin-Lechowska A, et al. Relationship between HER2 gene status and selected potential biological features related to trastuzumab resistance and its influence on survival of breast cancer patients undergoing trastuzumab adjuvant treatment. Onco Targets Ther. 2018;11:4525-4535. doi:10.2147/OTT.S166983

33. Stocker A, Hilbers ML, Gauthier C, et al. HER2/CEP17 ratios and clinical outcome in HER2-positive early breast cancer undergoing trastuzumab-containing therapy. PLoS One. 2016;11(7):e0159176. doi:10.1371/journal.pone.0159176

34. Perez EA, Reinholz MM, Hillman DW, et al. HER2 and chromosome 17 effect on patient outcome in the N9831 adjuvant trastuzumab trial. $J$ Clin Oncol. 2010;28(28):4307-4315. doi:10.1200/JCO.2009.26. 2154 
35. Fuchs EM, Kostler WJ, Horvat R, et al. High-level ERBB2 gene amplification is associated with a particularly short time-to-metastasis, but results in a high rate of complete response once trastuzumabbased therapy is offered in the metastatic setting. Int $J$ Cancer. 2014;135(1):224-231. doi:10.1002/ijc.28660

36. Kim JW, Kim JH, Im SA, et al. HER2/CEP17 ratio and HER2 immunohistochemistry predict clinical outcome after first-line trastuzumab plus taxane chemotherapy in patients with HER2 fluorescence in situ hybridization-positive metastatic breast cancer. Cancer Chemother Pharmacol. 2013;72(1):109-115. doi:10.1007/s00280-013-2174-1

37. Ellegard S, Veenstra C, Perez-Tenorio G, et al. ERBB2 and PTPN2 gene copy numbers as prognostic factors in HER2-positive metastatic breast cancer treated with trastuzumab. Oncol Lett. 2019;17(3):33713381. doi:10.3892/ol.2019.9998

38. Liang DH, Ensor JE, Liu ZB, et al. Cell-free DNA as a molecular tool for monitoring disease progression and response to therapy in breast cancer patients. Breast Cancer Res Treat. 2016;155(1):139149. doi:10.1007/s10549-015-3635-5
39. Molina R, Jo J, Filella X, et al. Serum levels of C-erbB-2 (HER-2/ neu) in patients with malignant and non-malignant diseases. Tumour Biol. 1997;18(3):188-196. doi:10.1159/000218029

40. Perrier A, Gligorov J, Lefevre G, Boissan M. The extracellular domain of Her2 in serum as a biomarker of breast cancer. Lab Invest. 2018;98(6):696-707. doi:10.1038/s41374-018-0033-8

41. Zhang Z, Li C, Fan H, et al. Prognostic value of baseline serum HER2 extracellular domain level with a cut-off value of $15 \mathrm{ng} / \mathrm{mL}$ in patients with breast cancer: a systematic review and meta-analysis. Breast Cancer Res Treat. 2018;172(3):513-521. doi:10.1007/s10549018-4942-4

42. Harris LN, Ismaila N, McShane LM, et al. Use of biomarkers to guide decisions on adjuvant systemic therapy for women with earlystage invasive breast cancer: American Society of Clinical Oncology clinical practice guideline. J Clin Oncol. 2016;34(10):1134-1150. doi:10.1200/JCO.2015.65.2289

\section{Publish your work in this journal}

OncoTargets and Therapy is an international, peer-reviewed, open access journal focusing on the pathological basis of all cancers, potential targets for therapy and treatment protocols employed to improve the management of cancer patients. The journal also focuses on the impact of management programs and new therapeutic agents and protocols on patient perspectives such as quality of life, adherence and satisfaction. The manuscript management system is completely online and includes a very quick and fair peer-review system, which is all easy to use. Visit http://www.dovepress.com/ testimonials.php to read real quotes from published authors. 